\title{
A Note on Conformable Double Laplace Transform and Singular Conformable Pseudoparabolic Equations
}

\author{
Hassan Eltayeb (iD) and Said Mesloub \\ Mathematics Department, College of Science, King Saud University, P.O. Box 2455, Riyadh 11451, Saudi Arabia \\ Correspondence should be addressed to Hassan Eltayeb; hgadain@ksu.edu.sa
}

Received 10 September 2019; Accepted 18 October 2019; Published 17 February 2020

Academic Editor: Maria Alessandra Ragusa

Copyright ( $\odot 2020$ Hassan Eltayeb and Said Mesloub. This is an open access article distributed under the Creative Commons Attribution License, which permits unrestricted use, distribution, and reproduction in any medium, provided the original work is properly cited.

In this work, we combine conformable double Laplace transform and Adomian decomposition method and present a new approach for solving singular one-dimensional conformable pseudoparabolic equation and conformable coupled pseudoparabolic equation. Furthermore, some examples are given to show the performance of the proposed method.

\section{Introduction}

Fractional partial differential equations have attracted much attention in applied sciences and engineering such as acoustics, control, and viscoelasticity. The parabolic equation appeared in different fields of applied mathematics, such as heat conduction and fluid mechanics (for instance, see $[1-4])$. The authors in $[5,6]$ studied the fractional diffusion equations problems by using the Adomian decomposition method and series expansion method. Many papers exist in the literature, which are related to conformable fractional derivative with its properties and applications $[7,8]$. This new method was quickly generalized by Katugampola $[9,10]$. The authors in [11] investigated existence and uniqueness theorems for sequential linear conformable fractional differential equations. The authors in [12] revisited the Grünwald Letnikov, Riemann-Liouville, and Caputo fractional derivatives and analysed under the light of the proposed criteria. The nonhomogeneous nonlocal theory has been presented based on conformable derivatives (CD) to study the critical point instability of micro/nanobeams under a distributed variable-pressure force (see [13]). The authors in [14] proposed a new fractional nonlocal model and its application in free vibration of Timoshenko and Euler-Bernoulli beams. Recently, several researchers applied the conformable Laplace transform method to solve different types of fractional differential equation (see $[15,16])$. Many exact solutions in various wave forms for the nonlinear conformable time-fractional parabolic equation with exponential nonlinearity are formally constructed in [17]. The goal of this paper is to investigate the solution of singular conformable fractional pseudoparabolic equation and conformable coupled pseudoparabolic equation by conformable double Laplace transform decomposition methods (CDLTDMs). Moreover, we are able to prove some theorems related to this work.

\subsection{Conformable Partial Derivatives}

Definition 1 (see [18]). Given a function $f(x, t): R \times$ $(0, \infty) \longrightarrow R$, the conformable space fractional partial derivative of order $\alpha$ of the function $f(x, t)$ is denoted by

$$
\frac{\partial^{\alpha}}{\partial x^{\alpha}} f(x, t)=\lim _{\varepsilon \longrightarrow 0} \frac{f\left(x+\varepsilon x^{1-\alpha}, t\right)-f(x, t)}{\varepsilon}, \quad x, t>0,0<\alpha \leq 1 .
$$

Definition 2 (see [18]). Given a function $f(x, t): R \times$ $(0, \infty) \longrightarrow R$, the conformable time partial derivative of order $\beta$ of the function $f(x, t)$ is defined as

$$
\frac{\partial^{\beta}}{\partial t^{\beta}} f(x, t)=\lim _{\sigma \longrightarrow 0} \frac{f\left(x, t+\sigma t^{1-\beta}\right)-f(x, t)}{\varepsilon}, \quad x, t>0,0<\beta \leq 1,
$$


where $\partial^{\alpha} / \partial x^{\alpha}$ and $\partial^{\beta} / \partial t^{\beta}$ are called the fractional derivatives of order $\alpha$ and $\beta$, respectively.

In Theorem 1, the connection between the conformable derivatives and the first derivative can be represented as follows.

Theorem 1. Let $\alpha, \beta \in(0,1]$ and $f(x, t)$ be $\alpha$ and $\beta$ differentiable at a point $x, t>0$.

Then,

$$
\begin{aligned}
& \frac{\partial^{\alpha} f(x, t)}{\partial x^{\alpha}}=x^{1-\alpha} \frac{\partial f(x, t)}{\partial x}, \\
& \frac{\partial^{\beta} f(x, t)}{\partial t^{\beta}}=t^{1-\beta} \frac{\partial f(x, t)}{\partial x} .
\end{aligned}
$$

Proof. By using definitions 1 and 2 and $h=\epsilon x^{1-\alpha}$ in equation (1), we have

$$
\begin{aligned}
\frac{\partial^{\alpha} f(x, t)}{\partial x^{\alpha}} & =\lim _{\varepsilon \longrightarrow 0} \frac{f\left(x+\varepsilon x^{1-\alpha}, t\right)-f(x, t)}{\varepsilon} \\
& =\lim _{h \longrightarrow 0} \frac{f(x+h, t)-f(x, t)}{h x^{\alpha-1}} \\
& =x^{1-\alpha} \lim _{h \longrightarrow 0} \frac{f(x+h, t)-f(x, t)}{h} \\
& =x^{1-\alpha} \frac{\partial f(x, t)}{\partial x} .
\end{aligned}
$$

Similarly, we prove equation (2).

In the next example, we introduce the conformable derivative of specific functions, by using Theorem 1 as follows.

Example 1. Let $\alpha, \beta \in(0,1]$ and $a, b, m, n, \lambda$, and $\mu \in \mathbb{R}$; then

(1) $\left(\partial^{\alpha} / \partial x^{\alpha}\right)(a u(x, t)+b v(x, t))=a\left(\partial^{\alpha} u(x, t) / \partial x^{\alpha}\right)+$ $b\left(\partial^{\alpha} v(x, t) / \partial x^{\alpha}\right)$

(2) $\left(\partial^{\alpha+\beta} / \partial x^{\alpha} \partial t^{\beta}\right)\left(x^{\mu} t^{\lambda}\right)=\mu \lambda x^{\kappa-\alpha} t^{\lambda-\beta}, \lambda, \mu \in \mathbb{R}$

(3) $\left(\partial^{\alpha} / \partial x^{\alpha}\right)\left(e^{\lambda\left(x^{\alpha} / \alpha\right)+\left(\tau t^{\beta} / \beta\right)}\right)=\lambda e^{\lambda\left(x^{\alpha} / \alpha\right)+\left(\tau t^{\beta} / \beta\right)}$, $\left(\partial^{\beta} / \partial t^{\beta}\right)\left(e^{\lambda\left(x^{\alpha} / \alpha\right)+\left(\tau t^{\beta} / \beta\right)}\right)=\tau e^{\lambda\left(x^{\alpha} / \alpha\right)+\left(\tau t^{\beta} / \beta\right)}$

(4) $\left(\partial^{\beta} / \partial t^{\beta}\right)\left(x^{\alpha} / \alpha\right)\left(t^{\beta} / \beta\right)=\left(x^{\alpha} / \alpha\right)$, $\left(\partial^{\beta} / \partial t^{\beta}\right)\left(x^{\alpha} / \alpha\right)^{n}\left(t^{\beta} / \beta\right)^{m}=m\left(x^{\alpha} / \alpha\right)^{n}\left(t^{\beta} / \beta\right)^{m-1}$

(5) $\left(\partial^{\alpha} / \partial x^{\alpha}\right)\left(x^{\alpha} / \alpha\right)^{n}\left(t^{\beta} / \beta\right)=n\left(x^{\alpha} / \alpha\right)^{n-1}\left(t^{\beta} / \beta\right)$, $\left(\partial^{\beta} / \partial t^{\beta}\right)\left(x^{\alpha} / \alpha\right)^{n}\left(t^{\beta} / \beta\right)^{m}=m\left(x^{\alpha} / \alpha\right)^{n}\left(t^{\beta} / \beta\right)^{m-1}$

(6) $\left(\partial^{\beta} / \partial t^{\beta}\right)\left(\sin \left(x^{\alpha} / \alpha\right) \sin \left(t^{\beta} / \beta\right)\right)=\sin \left(x^{\alpha} / \alpha\right) \cos$ $\left(t^{\beta} / \beta\right),\left(\partial^{\alpha} / \partial x^{\alpha}\right)\left(\sin a\left(x^{\alpha} / \alpha\right) \sin \left(t^{\beta} / \beta\right)\right)=$ $\sin \left(x^{\alpha} / \alpha\right) \cos \left(t^{\beta} / \beta\right)$

\section{Some Properties of the Conformable Laplace Transform}

Here, we work with the single conformable Laplace transform and conformable double Laplace transform (CDLT) which are defined, respectively as follows.
Definition 3 (see $[7,19,20]$ ). Let $f:[a, \infty) \longrightarrow \mathbb{R}$ and $0<\beta \leq 1$. Then, the fractional Laplace transform of order $\beta$ is defined by

$$
L_{t}^{\beta}(f(t))=\int_{0}^{\infty} e^{-s\left(t^{\beta} / \beta\right)} f(t) t^{\beta-1} \mathrm{~d} t .
$$

Definition 4 (see [21]). Let $u(x, t)$ be a piecewise continuous function on the interval $[a, \infty) \times[a, \infty)$ of exponential order. Consider for some $a, b \in \mathbb{R}$ sup $x, t>0$, $\left(|u(x, t)| / e^{\left(a x^{\alpha} / \alpha\right)+\left(b t^{\beta} / \beta\right)}\right)$ in these conditions (CDLT) is defined by

$$
L_{x}^{\alpha} L_{t}^{\beta}(u(x, t))=\int_{0}^{\infty} \int_{0}^{\infty} e^{-p\left(x^{\alpha} / \alpha\right)-s\left(t^{\beta} / \beta\right)} u(x, t) t^{\beta-1} x^{\alpha-1} \mathrm{~d} t \mathrm{~d} x,
$$

where $p, s \in \mathbb{C}, 0<\alpha, \beta \leq 1$, and the integrals are by means of conformable integral with respect to $t$ and $x$, respectively.

Theorem 2. If $L_{x}^{\alpha} L_{t}^{\beta}\left[f\left(x^{\alpha} / \alpha, t^{\beta} / \beta\right)\right]=F_{\alpha, \beta}(p, s)$, then

$$
\begin{aligned}
& L_{x}^{\alpha} L_{t}^{\beta}\left[f\left(\frac{x^{\alpha}}{\alpha}-\frac{\zeta^{\alpha}}{\alpha}, \frac{t^{\beta}}{\beta}-\frac{\eta^{\beta}}{\beta}\right) H\left(\frac{x^{\alpha}}{\alpha}-\frac{\zeta^{\alpha}}{\alpha}, \frac{t^{\beta}}{\beta}-\frac{\eta^{\beta}}{\beta}\right)\right] \\
& =e^{-p\left(\zeta^{\alpha} / \alpha\right)-s\left(\eta^{\beta} / \beta\right)} F_{\alpha, \beta}(p, s),
\end{aligned}
$$

where $H(x, t)$ is the Heaviside unit step function defined by $H\left(\left(x^{\alpha} / \alpha\right)-a,\left(t^{\beta} / \beta\right)-b\right)=1$ when $x>a$ and $t>b$ and $H\left(\left(x^{\alpha} / \alpha\right)-a,\left(t^{\beta} / \beta\right)-b\right)=0$ when $x<a$ and $t<b$.

Proof. By applying the definition of double conformable Laplace transform,

$$
\begin{aligned}
& L_{x}^{\alpha} L_{t}^{\beta}\left[f\left(\frac{x^{\alpha}}{\alpha}-\frac{\zeta^{\alpha}}{\alpha}, \frac{t^{\beta}}{\beta}-\frac{\eta^{\beta}}{\beta}\right) H\left(\frac{x^{\alpha}}{\alpha}-\frac{\zeta^{\alpha}}{\alpha}, \frac{t^{\beta}}{\beta}-\frac{\eta^{\beta}}{\beta}\right)\right] \\
& =\int_{0}^{\infty} \int_{0}^{\infty} e^{-p\left(x^{\alpha} / \alpha\right)-s\left(t^{\beta} / \beta\right)} f\left(\frac{x^{\alpha}}{\alpha}-\frac{\zeta^{\alpha}}{\alpha}, \frac{t^{\beta}}{\beta}-\frac{\eta^{\beta}}{\beta}\right) \\
& \cdot H\left(\frac{x^{\alpha}}{\alpha}-\frac{\zeta^{\alpha}}{\alpha}, \frac{t^{\beta}}{\beta}-\frac{\eta^{\beta}}{\beta}\right) t^{\beta-1} x^{\alpha-1} \mathrm{~d} t \mathrm{~d} x \\
& =\int_{\zeta^{\alpha} / \alpha}^{\infty} \int_{\eta^{\beta} / \beta}^{\infty} e^{-p\left(x^{\alpha} / \alpha\right)-s\left(t^{\beta} / \beta\right)} f\left(\frac{x^{\alpha}}{\alpha}-\frac{\zeta^{\alpha}}{\alpha}, \frac{t^{\beta}}{\beta}-\frac{\eta^{\beta}}{\beta}\right) t^{\beta-1} x^{\alpha-1} \mathrm{~d} t \mathrm{~d} x,
\end{aligned}
$$

which is, by putting $\left(x^{\alpha} / \alpha\right)-\left(\zeta^{\alpha} / \alpha\right)=\tau^{\alpha} / \alpha,\left(t^{\beta} / \beta\right)-\left(\eta^{\beta} / \beta\right)$ $=\nu^{\beta} / \beta$, we have

$$
\begin{aligned}
& L_{x}^{\alpha} L_{t}^{\beta}\left[f\left(\frac{x^{\alpha}}{\alpha}-\frac{\zeta^{\alpha}}{\alpha}, \frac{t^{\beta}}{\beta}-\frac{\eta^{\beta}}{\beta}\right) H\left(\frac{x^{\alpha}}{\alpha}-\frac{\zeta^{\alpha}}{\alpha}, \frac{t^{\beta}}{\beta}-\frac{\eta^{\beta}}{\beta}\right)\right] \\
& =e^{-p\left(\zeta^{\alpha} / \alpha\right)-s\left(\eta^{\beta} / \beta\right)} \int_{0}^{\infty} \int_{0}^{\infty} e^{-p\left(\tau^{\alpha / \alpha}\right)-s\left(v^{\beta} / \beta\right)} f\left(\frac{\tau^{\alpha}}{\alpha}, \frac{v^{\beta}}{\beta}\right) v^{\beta-1} \tau^{\alpha-1} \mathrm{~d} v \mathrm{~d} \tau \\
& =e^{-p\left(\zeta^{\alpha} / \alpha\right)-s\left(\eta^{\beta} \beta / \beta\right.} F_{\alpha, \beta}(p, s) .
\end{aligned}
$$


In the next example, we reported that some conformable Laplace transforms of definite functions are important in this study.

\section{Example 2}

(1) $L_{x}^{\alpha} L_{t}^{\beta}\left[\left(x^{\alpha} / \alpha\right)^{n} \quad\left(t^{\beta} / \beta\right)^{m}\right]=L_{x} L_{t}\left[(x)^{n}(t)^{m}\right]=n ! m ! /$ $p^{n+1} s^{m+1}$, where $m$ and $n$ are positive integers

(2) $L_{x}^{\alpha} L_{t}^{\beta}\left[e^{\lambda\left(x^{\alpha} / \alpha\right)+\left(\tau t^{\beta} / \beta\right)}\right]=L_{x} L_{t}\left[e^{\lambda x+\tau t}\right]=1 /((p-\lambda)$ $(s-\tau))$

(3) $L_{x}^{\alpha} L_{t}^{\beta}\left[\sin \left(\lambda\left(x^{\alpha} / \alpha\right)\right) \sin \left(\tau\left(t^{\beta} / \beta\right)\right)\right]=L_{x} L_{t}[\sin$ $(x) \cos (t)]=1 /\left(\left(p^{2}+\lambda^{2}\right)\left(s^{2}+\tau^{2}\right)\right)$

Theorem 3. Let $f$ be piecewise continuous on $[a, \infty) \times[a, \infty)$; the $(C D L T)$ of the conformable partial derivatives of orders $\alpha$-th and $\beta$-th, $\partial^{\alpha} u / \partial x^{\alpha}, \partial^{\beta} u / \partial t^{\beta}, \partial^{2 \alpha} u / \partial x^{2 \alpha}$, and $\partial^{2 \beta} u / \partial t^{2 \beta}$ is given by

$$
\begin{aligned}
L_{x}^{\alpha} L_{t}^{\beta}\left(\frac{\partial^{\alpha} u}{\partial x^{\alpha}}\right) & =p U(p, s)-U(0, s), \\
L_{x}^{\alpha} L_{t}^{\beta}\left(\frac{\partial^{\beta} u}{\partial t^{\beta}}\right) & =s U(p, s)-U(p, 0), \\
L_{x}^{\alpha} L_{t}^{\beta}\left(\frac{\partial^{2 \alpha} u}{\partial x^{2 \alpha}}\right) & =p^{2} U(p, s)-p U(0, s)-U_{x}(0, s), \\
L_{x}^{\alpha} L_{t}^{\beta}\left(\frac{\partial^{\beta} u}{\partial t^{\beta}}\right) & =s^{2} U(p, s)-s U(p, 0)-U_{t}(p, 0) .
\end{aligned}
$$

Proof. By using definition (CDLT) for $\partial^{\alpha} u / \partial x^{\alpha}$, we have

$$
\begin{aligned}
L_{x}^{\alpha} L_{t}^{\beta}\left(\frac{\partial^{\alpha} u}{\partial x^{\alpha}}\right) & =\int_{0}^{\infty} \int_{0}^{\infty} e^{-p\left(x^{\alpha} / \alpha\right)-s\left(t^{\beta} / \beta\right)} \frac{\partial^{\alpha} u}{\partial x^{\alpha}} t^{\beta-1} x^{\alpha-1} \mathrm{~d} t \mathrm{~d} x \\
& =\int_{0}^{\infty} e^{-s\left(t^{\beta} / \beta\right)} t^{\beta-1}\left(\int_{0}^{\infty} e^{-p\left(x^{\alpha} / \alpha\right)} \frac{\partial^{\alpha} u}{\partial x^{\alpha}} x^{\alpha-1} \mathrm{~d} x\right) \mathrm{d} t .
\end{aligned}
$$

By applying Theorem $1, \partial^{\alpha} u / \partial x^{\alpha}=x^{1-\alpha}(\partial u(x, t) / \partial x)$ equation (13) becomes

$$
L_{x}^{\alpha} L_{t}^{\beta}\left(\frac{\partial^{\alpha} u}{\partial x^{\alpha}}\right)=\int_{0}^{\infty} e^{-s\left(t^{\beta} / \beta\right)} t^{\beta-1}\left(\int_{0}^{\infty} e^{-p\left(x^{\alpha} / \alpha\right)} \frac{\partial u(x, t)}{\partial x} \mathrm{~d} x\right) d t .
$$

The integral inside bracket given by

$$
\int_{0}^{\infty} e^{-p\left(x^{\alpha} / \alpha\right)} \frac{\partial u(x, t)}{\partial x} \mathrm{~d} x=p U(p, t)-U(0, t) .
$$
obtain

By substituting equation (15) into equation (14), we

$$
L_{x}^{\alpha} L_{t}^{\beta}\left(\frac{\partial^{\alpha} u}{\partial x^{\alpha}}\right)=p U(p, s)-U(0, s) .
$$

In the same manner, the (CDLT) of $\partial^{\beta} u / \partial t^{\beta}, \partial^{2 \alpha} u / \partial x^{2 \alpha}$, and $\partial^{2 \beta} u / \partial t^{2 \beta}$ can be obtained.
Double Laplace transform of the function $\left(x^{\alpha} / \alpha\right)^{n}\left(\partial^{\beta} f / \partial t^{\beta}\right)$ and $\left(x^{\alpha} / \alpha\right) f(x, t)$ are studied in the next theorem.

Theorem 4. If the (CDLT) of the conformable partial derivatives $\left(\partial^{\beta} / \partial t^{\beta}\right) u$ is given by equation (11), then double Laplace transform of $\left(x^{\alpha} / \alpha\right)^{n}\left(\partial^{\beta} / \partial t^{\beta}\right) u(x, t)$ and $\left(x^{\alpha} / \alpha\right) u(x, t)$ are given by

$$
\begin{aligned}
& (-1)^{n} \frac{\mathrm{d}^{n}}{\mathrm{~d} p^{n}}\left(L_{x}^{\alpha} L_{t}^{\beta}[u(x, t)]\right)=L_{x}^{\alpha} L_{t}^{\beta}\left[\left(\frac{x^{\alpha}}{\alpha}\right)^{n} u(x, t)\right], \\
& (-1)^{n} \frac{\mathrm{d}^{n}}{\mathrm{~d} p^{n}}\left(L_{x}^{\alpha} L_{t}^{\beta}\left[\frac{\partial^{\beta} u}{\partial t^{\beta}}\right]\right)=L_{x}^{\alpha} L_{t}^{\beta}\left[\left(\frac{x^{\alpha}}{\alpha}\right)^{n} \frac{\partial^{\beta} u}{\partial t^{\beta}}\right],
\end{aligned}
$$

where $n=1,2,3, \ldots$

Proof. By applying the nth derivative with respect to $p$ for both sides of equation (6), we get equation (17) as follows:

$$
\begin{aligned}
\frac{\mathrm{d}^{n}}{\mathrm{~d} p^{n}}\left(L_{x}^{\alpha} L_{t}^{\beta}[u(x, t)]\right)= & \int_{0}^{\infty} \int_{0}^{\infty} \frac{\mathrm{d}^{n}}{\mathrm{~d} p^{n}}\left(e^{-p\left(x^{\alpha} / \alpha\right)-s\left(t^{\beta} / \beta\right)} u(x, t)\right) \\
& \cdot t^{\beta-1} x^{\alpha-1} \mathrm{~d} t \mathrm{~d} x \\
= & (-1)^{n} \int_{0}^{\infty} \int_{0}^{\infty}\left(\frac{x^{\alpha}}{\alpha}\right)^{n} e^{-p\left(x^{\alpha} / \alpha\right)-s\left(t^{\beta} / \beta\right)} \\
& \cdot t^{\beta-1} x^{\alpha-1} u(x, t) \mathrm{d} t \mathrm{~d} x \\
= & (-1)^{n} L_{x}^{\alpha} L_{t}^{\beta}\left[\left(\frac{x^{\alpha}}{\alpha}\right)^{n} u(x, t)\right] .
\end{aligned}
$$

We obtain

$$
(-1)^{n} \frac{\mathrm{d}^{n}}{\mathrm{~d} p^{n}}\left(L_{x}^{\alpha} L_{t}^{\beta}[u(x, t)]\right)=L_{x}^{\alpha} L_{t}^{\beta}\left[\left(\frac{x^{\alpha}}{\alpha}\right)^{n} u(x, t)\right] .
$$

Similarly, we can prove equation (18).

\section{Singular One-Dimensional Conformable Fractional Pseudoparabolic Equation}

The conformable double Laplace decomposition methods (CDLTDMs) are an efficient technique which is used to obtain the solution linear and nonlinear singular pseudoparabolic equation.

Problem. We consider $0<\alpha \leq 1$ and $0<\beta \leq 1$ as singular one-dimensional pseudoparabolic equations with initial conditions in the form

$$
\frac{\partial^{\beta} u}{\partial t^{\beta}}-\frac{\alpha}{x^{\alpha}} \frac{\partial^{\alpha}}{\partial x^{\alpha}}\left(\frac{x^{\alpha}}{\alpha} \frac{\partial^{\alpha}}{\partial x^{\alpha}} u\right)-\frac{\alpha}{x^{\alpha}} \frac{\partial^{\alpha+\beta}}{\partial x^{\alpha} \partial t^{\beta}}\left(\frac{x^{\alpha}}{\alpha} \frac{\partial^{\alpha}}{\partial x^{\alpha}} u\right)=f(x, t),
$$

subject to

$$
u(x, 0)=f_{1}(x),
$$

where, the term, $\left(\alpha / x^{\alpha}\right)\left(\partial^{\alpha} u / \partial x^{\alpha}\right)\left(\left(x^{\alpha} / \alpha\right)\left(\partial^{\alpha} / \partial x^{\alpha}\right)\right)$ is called conformable Bessel's operator and $f(x, t)$ and $f_{1}(x)$ are 
known functions. In order to solve equation (21), we apply the following steps:

Step 1: multiplying equation (21) by $x^{\alpha} / \alpha$ :

$$
\begin{gathered}
\frac{x^{\alpha}}{\alpha} \frac{\partial^{\beta} u}{\partial t^{\beta}}-\frac{\partial^{\alpha}}{\partial x^{\alpha}}\left(\frac{x^{\alpha}}{\alpha} \frac{\partial^{\alpha}}{\partial x^{\alpha}} u\right)-\frac{\partial^{\alpha+\beta}}{\partial x^{\alpha} \partial t^{\beta}}\left(\frac{x^{\alpha}}{\alpha} \frac{\partial^{\alpha}}{\partial x^{\alpha}} u\right) \\
=\frac{x^{\alpha}}{\alpha} f(x, t) .
\end{gathered}
$$

Step 2: using Lemma 1 and equation (18) for equations in step 1 and single conformable Laplace transform for equation (22), we obtain

$$
\begin{aligned}
\frac{\mathrm{d}}{\mathrm{d} p} U(p, s)= & \frac{1}{s} \frac{\mathrm{d}}{\mathrm{d} p} L_{x}^{\alpha}\left[f_{1}(x)\right]+\frac{1}{s} \frac{\mathrm{d}}{\mathrm{d} p}\left[L_{x}^{\alpha} L_{t}^{\beta}[f(x, t)]\right] \\
& -\frac{1}{s} L_{x}^{\alpha} L_{t}^{\beta}\left[\frac{\partial^{\alpha}}{\partial x^{\alpha}}\left(\frac{x^{\alpha}}{\alpha} \frac{\partial^{\alpha}}{\partial x^{\alpha}} u\right)\right. \\
& \left.+\frac{\partial^{\alpha+\beta}}{\partial x^{\alpha} \partial t^{\beta}}\left(\frac{x^{\alpha}}{\alpha} \frac{\partial^{\alpha}}{\partial x^{\alpha}} u\right)\right]
\end{aligned}
$$

where the symbol $L_{x}^{\alpha} L_{t}^{\beta}$ indicates (CDLT) with respect to $x$ and $t$.
Step 3: applying the integral for both sides of equation (24), from 0 to $p$ with respect to $p$, we have

$$
\begin{aligned}
U(p, s)= & \frac{1}{s} \int_{0}^{p}\left(\frac{\mathrm{d}}{\mathrm{d} p} L_{x}^{\alpha}\left[f_{1}(x)\right]\right) \mathrm{d} p-\frac{1}{s} \int_{0}^{p} L_{x}^{\alpha} L_{t}^{\beta}\left[\frac { \partial ^ { \alpha } } { \partial x ^ { \alpha } } \left(\frac{x^{\alpha}}{\alpha}\right.\right. \\
& \left.\left.\cdot \frac{\partial^{\alpha}}{\partial x^{\alpha}} u\right)+\frac{\partial^{\alpha+\beta}}{\partial x^{\alpha} \partial t^{\beta}}\left(\frac{x^{\alpha}}{\alpha} \frac{\partial^{\alpha}}{\partial x^{\alpha}} u\right)\right] \mathrm{d} p \\
& +\frac{1}{s} \int_{0}^{p}\left(\frac{\mathrm{d}}{\mathrm{d} p}\left[L_{x}^{\alpha} L_{t}^{\beta}[f(x, t)]\right]\right) \mathrm{d} p .
\end{aligned}
$$

Step 4: next, the (CDLTDM) consists of representing the solution of the singular pseudoparabolic equation as $u(x, t)$ by the infinite series

$$
u(x, t)=\sum_{n=0}^{\infty} u_{n}(x, t)
$$

Step 5: working with the double Laplace transform on both sides of equation (25) and using equation (26), we receive

$$
\begin{aligned}
\sum_{n=0}^{\infty} u_{n}(x, t)= & f_{1}(x)+L_{p}^{-1} L_{s}^{-1}\left[\frac{1}{s} \int_{0}^{p}\left(\frac{\mathrm{d}}{\mathrm{d} p}\left(L_{x}^{\alpha} L_{t}^{\beta}[f(x, t)]\right)\right) \mathrm{d} p\right]-L_{p}^{-1} L_{s}^{-1}\left[\frac { 1 } { s } \int _ { 0 } ^ { p } \left(L _ { x } ^ { \alpha } L _ { t } ^ { \beta } \left[\frac { \partial ^ { \alpha } } { \partial x ^ { \alpha } } \left(\frac{x^{\alpha}}{\alpha} \frac{\partial^{\alpha}}{\partial x^{\alpha}}\right.\right.\right.\right. \\
& \left.\left.\left.\left.\cdot\left(\sum_{n=0}^{\infty} u_{n}(x, t)\right)\right)\right]\right) \mathrm{d} p\right]-L_{p}^{-1} L_{s}^{-1}\left[\frac{1}{s} \int_{0}^{p}\left(L_{x}^{\alpha} L_{t}^{\beta}\left[\frac{\partial^{\alpha+\beta}}{\partial x^{\alpha} \partial t^{\beta}}\left(\frac{x^{\alpha}}{\alpha} \frac{\partial^{\alpha}}{\partial x^{\alpha}}\left(\sum_{n=0}^{\infty} u_{n}(x, t)\right)\right)\right]\right) \mathrm{d} p\right]
\end{aligned}
$$

We define the following recursive formula:

$u_{0}(x, t)=f_{1}(x)+L_{p}^{-1} L_{s}^{-1}\left[\frac{1}{s} \int_{0}^{p}\left(\frac{\mathrm{d}}{\mathrm{d} p}\left(L_{x}^{\alpha} L_{t}^{\beta}[f(x, t)]\right)\right) \mathrm{d} p\right]$.

The rest of the terms can be written as follows:

$$
\begin{aligned}
u_{n+1}(x, t)= & -L_{p}^{-1} L_{s}^{-1}\left[\frac{1}{s} \int_{0}^{p}\left(L_{x}^{\alpha} L_{t}^{\beta}\left[\frac{\partial^{\alpha}}{\partial x^{\alpha}}\left(\frac{x^{\alpha}}{\alpha} \frac{\partial^{\alpha}}{\partial x^{\alpha}}\left(\sum_{n=0}^{\infty} u_{n}(x, t)\right)\right)\right]\right) \mathrm{d} p\right]-L_{p}^{-1} L_{s}^{-1} \\
& \cdot\left[\frac{1}{s} \int_{0}^{p}\left(L_{x}^{\alpha} L_{t}^{\beta}\left[\frac{\partial^{\alpha+\beta}}{\partial x^{\alpha} \partial t^{\beta}}\left(\frac{x^{\alpha}}{\alpha} \frac{\partial^{\alpha}}{\partial x^{\alpha}}\left(\sum_{n=0}^{\infty} u_{n}(x, t)\right)\right)\right] \mathrm{d} p\right],\right.
\end{aligned}
$$

where $L_{p}^{-1} L_{s}^{-1}$ indicates double inverse Laplace transform with respect to $p$ and $s$.

Here, we assume that double inverse Laplace transform with respect to $p$ and $s$ exists for each terms in equations
(28) and (29). To confirm our method, we solve the next example.

Example 3. Consider the following nonhomogeneous form of a singular one-dimensional pseudoparabolic equation: 


$$
\begin{aligned}
\frac{\partial^{\beta} u}{\partial t^{\beta}}-\frac{\alpha}{x^{\alpha}} \frac{\partial^{\alpha}}{\partial x^{\alpha}}\left(\frac{x^{\alpha}}{\alpha} \frac{\partial^{\alpha}}{\partial x^{\alpha}} u\right)-\frac{\alpha}{x^{\alpha}} \frac{\partial^{\alpha+\beta} u}{\partial x^{\alpha} \partial t^{\beta}}\left(\frac{x^{\alpha}}{\alpha} \frac{\partial^{\alpha}}{\partial x^{\alpha}} u\right) \\
=-\left(\frac{x^{\alpha}}{\alpha}\right)^{2} \sin \left(\frac{t^{\beta}}{\beta}\right)+4 \sin \left(\frac{t^{\beta}}{\beta}\right)-4 \cos \left(\frac{t^{\beta}}{\beta}\right), \\
0 \leq x<\infty, 0 \leq t<\infty, \\
0<\alpha \leq 1,0<\beta \leq 1,
\end{aligned}
$$

with the condition

$$
u(x, t)=x^{2} .
$$

By applying the above steps and Theorem 1, we obtain

$$
\begin{aligned}
u_{0}(x, t)= & \left(\frac{x^{\alpha}}{\alpha}\right)^{2} \cos \left(\frac{t^{\beta}}{\beta}\right)-4 \cos \left(\frac{t^{\beta}}{\beta}\right)-4 \sin \left(\frac{t^{\beta}}{\beta}\right)+4, \\
u_{n+1}(x, t)= & -L_{p}^{-1} L_{s}^{-1}\left[\frac{1}{s} \int_{0}^{p}\left(L_{x}^{\alpha} L_{t}^{\beta}\left[\frac{\partial^{\alpha}}{\partial x^{\alpha}}\left(\frac{x^{\alpha}}{\alpha} \frac{\partial^{\alpha} u_{n}}{\partial x^{\alpha}}\right)\right]\right) \mathrm{d} p\right] \\
& -L_{p}^{-1} L_{s}^{-1}\left[\frac{1}{s} \int_{0}^{p}\left(L_{x}^{\alpha} L_{t}^{\beta}\left[\frac{\partial^{\alpha+\beta}}{\partial x^{\alpha} \partial t^{\beta}}\left(\frac{x^{\alpha}}{\alpha} \frac{\partial^{\alpha} u_{n}}{\partial x^{\alpha}}\right)\right]\right) \mathrm{d} p\right] .
\end{aligned}
$$

Based on the (CDLTDM), we obtain

$$
\begin{aligned}
u_{1}(x, t)= & -L_{p}^{-1} L_{s}^{-1}\left[\frac { 1 } { s } \int _ { 0 } ^ { p } \left(L _ { x } L _ { t } \left[\frac{\partial^{\alpha}}{\partial x^{\alpha}}\left(\frac{x^{\alpha}}{\alpha} \frac{\partial^{\alpha}}{\partial x^{\alpha}} u_{0}\right)\right.\right.\right. \\
& \left.\left.\left.+\frac{\partial^{\alpha+\beta}}{\partial x^{\alpha} \partial t^{\beta}}\left(\frac{x^{\alpha}}{\alpha} \frac{\partial^{\alpha}}{\partial x^{\alpha}} u_{0}\right)\right]\right) \mathrm{d} p\right] \\
= & -4+4 \cos \left(\frac{t^{\beta}}{\beta}\right)+4 \sin \left(\frac{t^{\beta}}{\beta}\right) .
\end{aligned}
$$

In a similar manner, we obtain that

$$
\begin{aligned}
u_{2}(x, t)= & -L_{p}^{-1} L_{s}^{-1}\left[\frac { 1 } { s } \int _ { 0 } ^ { p } \left(L _ { x } L _ { t } \left[\frac{\partial^{\alpha}}{\partial x^{\alpha}}\left(\frac{x^{\alpha}}{\alpha} \frac{\partial^{\alpha}}{\partial x^{\alpha}} u_{1}\right)\right.\right.\right. \\
& \left.\left.\left.+\frac{\partial^{\alpha+\beta}}{\partial x^{\alpha} \partial t^{\beta}}\left(\frac{x^{\alpha}}{\alpha} \frac{\partial^{\alpha}}{\partial x^{\alpha}} u_{1}\right)\right]\right) \mathrm{d} p\right] \\
= & 0 \\
u_{3}= & 0, u_{4}=0, \ldots
\end{aligned}
$$

By adding all the terms, we get

$$
u(x, t)=u_{0}(x, t)+u_{1}(x, t)+u_{2}(x, t)+\cdots .
$$

Thus, the exact solution is obtained as follows:

$$
u(x, t)=\left(\frac{x^{\alpha}}{\alpha}\right)^{2} \cos \left(\frac{t^{\beta}}{\beta}\right)
$$

By taking $\alpha=1$ and $\beta=1$, the fractional solution becomes

$$
u(x, t)=x^{2} \cos (t) \text {. }
$$

Problem. Consider the following nonlinear singular onedimensional pseudoparabolic equation:

$$
\begin{array}{r}
\frac{\partial^{\beta} u}{\partial t^{\beta}}-\frac{\alpha}{x^{\alpha}} \frac{\partial^{\alpha}}{\partial x^{\alpha}}\left(\frac{x^{\alpha}}{\alpha} \frac{\partial^{\alpha} u}{\partial x^{\alpha}}\right)-\frac{\alpha}{x^{\alpha}} \frac{\partial^{\alpha+\beta}}{\partial x^{\alpha} \partial t^{\beta}}\left(\frac{x^{\alpha}}{\alpha} \frac{\partial^{\alpha} u}{\partial x^{\alpha}}\right) \\
=-2 u \frac{\partial^{\alpha} u}{\partial x^{\alpha}}+\frac{x^{\alpha}}{\alpha}\left(\frac{\partial^{\alpha} u}{\partial x^{\alpha}}\right)^{2}+f(x, t), \\
0<\alpha \leq 1,0<\beta \leq 1, \\
0 \leq x<\infty, 0 \leq t<\infty,
\end{array}
$$

subject to

$$
u(x, 0)=f_{1}(x) .
$$

Using our method, we get

$$
u_{0}(x, t)=f_{1}(x)+L_{p}^{-1} L_{s}^{-1}\left[\frac{1}{s} \int_{0}^{p}\left(\frac{\mathrm{d}}{\mathrm{d} p}\left(L_{x}^{\alpha} L_{t}^{\beta}[f(x, t)]\right)\right) \mathrm{d} p\right] .
$$

The rest of the terms are given by

$$
\begin{aligned}
u_{n+1}(x, t)= & -L_{p}^{-1} L_{s}^{-1}\left[\frac{1}{s} \int_{0}^{p}\left(L_{x}^{\alpha} L_{t}^{\beta}\left[\frac{\partial^{\alpha}}{\partial x^{\alpha}}\left(\frac{x^{\alpha}}{\alpha} \frac{\partial^{\alpha} u_{n}}{\partial x^{\alpha}}\right)\right]\right) \mathrm{d} p\right] \\
& -L_{p}^{-1} L_{s}^{-1}\left[\frac{1}{s} \int_{0}^{p}\left(L_{x}^{\alpha} L_{t}^{\beta}\left[\frac{\partial^{\alpha+\beta}}{\partial x^{\alpha} \partial t^{\beta}}\left(\frac{x^{\alpha}}{\alpha} \frac{\partial^{\alpha} u_{n}}{\partial x^{\alpha}}\right)\right]\right) \mathrm{d} p\right] \\
& +L_{p}^{-1} L_{s}^{-1}\left[\frac{1}{s} \int_{0}^{p}\left(L_{x}^{\alpha} L_{t}^{\beta}\left[\left(2 \frac{x^{\alpha}}{\alpha} A_{n}\right)\right]\right) \mathrm{d} p\right] \\
& -L_{p}^{-1} L_{s}^{-1}\left[\frac{1}{s} \int_{0}^{p}\left(L_{x}^{\alpha} L_{t}^{\beta}\left[\left(\left(\frac{x^{\alpha}}{\alpha}\right)^{2} B_{n}\right)\right]\right) \mathrm{d} p\right],
\end{aligned}
$$

where $A_{n}$ and $B_{n}$ are the so-called Adomian polynomials, given by

$$
\begin{aligned}
& A_{n}=\sum_{n=0}^{\infty} u_{n} u_{n x}, \\
& B_{n}=\sum_{n=0}^{\infty}\left(u_{n x}\right)^{2} .
\end{aligned}
$$

The nonlinear terms $u u_{x}$ and $\left(u_{x}\right)^{2}$ are represented as

$$
\begin{aligned}
& A_{0}=u_{0} u_{0 x}, \\
& A_{1}=u_{0} u_{1 x}+u_{1} u_{0 x}, \\
& A_{2}=u_{0} u_{2 x}+u_{1} u_{1 x}+u_{2} u_{0 x}, \\
& A_{3}=u_{0} u_{3 x}+u_{1} u_{2 x}+u_{2} u_{1 x}+u_{3} u_{0 x}, \\
& B_{0}=\left(u_{0 x}\right)^{2}, \\
& B_{1}=2 u_{0 x} u_{1 x}, \\
& B_{2}=2 u_{0 x} u_{2 x}+\left(u_{1 x}\right)^{2}, \\
& B_{3}=2 u_{0 x} u_{3 x}+2 u_{1 x} u_{2 x} .
\end{aligned}
$$


To illustrate this method for nonlinear problem, we consider the following example.

Example 4. Consider the following nonlinear pseudoparabolic equation:

$$
\begin{array}{r}
\frac{\partial^{\beta} u}{\partial t^{\beta}}-\frac{\alpha}{x^{\alpha}} \frac{\partial^{\alpha}}{\partial x^{\alpha}}\left(\frac{x^{\alpha}}{\alpha} \frac{\partial^{\alpha} u}{\partial x^{\alpha}}\right)-\frac{\alpha}{x^{\alpha}} \frac{\partial^{\alpha+\beta}}{\partial x^{\alpha} \partial t^{\beta}}\left(\frac{x^{\alpha}}{\alpha} \frac{\partial^{\alpha} u}{\partial x^{\alpha}}\right) \\
=-2 u \frac{\partial^{\alpha} u}{\partial x^{\alpha}}+\frac{x^{\alpha}}{\alpha}\left(\frac{\partial^{\alpha} u}{\partial x^{\alpha}}\right)^{2}-\left(\frac{x^{\alpha}}{\alpha}\right)^{2} e^{-\left(t^{\beta} / \beta\right),} \\
0 \leq x<\infty, 0 \leq t<\infty, \\
0<\alpha \leq 1,0<\beta \leq 1,
\end{array}
$$

subject to

$$
u(x, 0)=\left(\frac{x^{\alpha}}{\alpha}\right)^{2} .
$$

By applying the aforesaid conformable double Laplace decomposition method and Theorem 1, we have

$$
\begin{aligned}
& u_{0}(x, t)=\left(\frac{x^{\alpha}}{\alpha}\right)^{2} e^{-\left(t^{\beta} \beta\right)}, \\
& u_{1}(x, t)=-L_{p}^{-1} L_{s}^{-1}\left[\frac { 1 } { s } \int _ { 0 } ^ { p } \left(L _ { x } ^ { \alpha } L _ { t } ^ { \beta } \left[\frac{\partial^{\alpha}}{\partial x^{\alpha}}\left(\frac{x^{\alpha}}{\alpha} \frac{\partial^{\alpha} u_{n}}{\partial x^{\alpha}}\right)+\frac{\partial^{\alpha+\beta}}{\partial x^{\alpha} \partial t^{\beta}}\right.\right.\right. \\
&\left.\left.\left.\cdot\left(\frac{x^{\alpha}}{\alpha} \frac{\partial^{\alpha} u_{n}}{\partial x^{\alpha}}\right)\right]\right) \mathrm{d} p\right]+L_{p}^{-1} L_{s}^{-1}\left[\frac { 1 } { s } \int _ { 0 } ^ { p } \left(L_{x}^{\alpha} L_{t}^{\beta}\right.\right. \\
&\left.\left.\cdot\left[\left(2 \frac{x^{\alpha}}{\alpha} A_{n}\right)-\left(\frac{x^{\alpha}}{\alpha}\right)^{2} B_{n}\right]\right) \mathrm{d} p\right]=0 .
\end{aligned}
$$

Proceeding in a similar manner, we have

$$
\begin{aligned}
& u_{2}=0, \\
& u_{3}=0, \\
& u_{4}=0, \ldots
\end{aligned}
$$

So according to equation (26), we have

$$
u(x, t)=\left(\frac{x^{\alpha}}{\alpha}\right)^{2} e^{-\left(t^{\beta} / \beta\right)},
$$

which is the exact solution of equation (44).

\section{Conformable Double Laplace Transform Method and Singular Conformable Coupled Pseudoparabolic Equation}

The purpose of this part is to examine the use of the (CDLTDM) for the linear one-dimensional conformable coupled pseudoparabolic equation. We consider the following conformable coupled pseudoparabolic equations: $\frac{\partial^{\beta} u}{\partial t^{\beta}}-\left(\frac{\alpha}{x^{\alpha}}\right) \frac{\partial^{\alpha}}{\partial x^{\alpha}}\left(\frac{x^{\alpha}}{\alpha} \frac{\partial^{\alpha} u}{\partial x^{\alpha}}\right)-\frac{\alpha}{x^{\alpha}} \frac{\partial^{\alpha+\beta}}{\partial x^{\alpha} \partial t^{\beta}}\left(\frac{x^{\alpha}}{\alpha} \frac{\partial^{\alpha} u}{\partial x^{\alpha}}\right)+\zeta v=f(x, t)$,

$\frac{\partial^{\beta} v}{\partial t^{\beta}}-\left(\frac{\alpha}{x^{\alpha}}\right) \frac{\partial^{\alpha}}{\partial x^{\alpha}}\left(\frac{x^{\alpha}}{\alpha} \frac{\partial^{\alpha} v}{\partial x^{\alpha}}\right)-\frac{\alpha}{x^{\alpha}} \frac{\partial^{\alpha+\beta}}{\partial x^{\alpha} \partial t^{\beta}}\left(\frac{x^{\alpha}}{\alpha} \frac{\partial^{\alpha} v}{\partial x^{\alpha}}\right)+\zeta u=g(x, t)$,

with conditions

$$
\begin{aligned}
& u(x, 0)=f_{1}(x), \\
& v(x, 0)=g_{1}(x),
\end{aligned}
$$

where $f(x, t), g(x, t), f_{1}(x)$, and $g_{1}(x)$ are the known functions and $\zeta$ is the coupling parameter. One can get the solution of equation (49), by using (CDLTDM); this method consists of the following steps:

(1) Multiply both sides of equation (49) by $x^{\alpha} / \alpha$ leading to the following equation:

$$
\begin{aligned}
& \frac{x^{\alpha}}{\alpha} \frac{\partial^{\beta} u}{\partial t^{\beta}}-\frac{\partial^{\alpha}}{\partial x^{\alpha}}\left(\frac{x^{\alpha}}{\alpha} \frac{\partial^{\alpha} u}{\partial x^{\alpha}}\right)-\frac{\partial^{\alpha+\beta}}{\partial x^{\alpha} \partial t^{\beta}}\left(\frac{x^{\alpha}}{\alpha} \frac{\partial^{\alpha} u}{\partial x^{\alpha}}\right) \\
& +\zeta \frac{x^{\alpha}}{\alpha} v=\frac{x^{\alpha}}{\alpha} f(x, t), \\
& \frac{x^{\alpha}}{\alpha} \frac{\partial^{\beta} v}{\partial t^{\beta}}-\frac{\partial^{\alpha}}{\partial x^{\alpha}}\left(\frac{x^{\alpha}}{\alpha} \frac{\partial^{\alpha} v}{\partial x^{\alpha}}\right)-\frac{\partial^{\alpha+\beta}}{\partial x^{\alpha} \partial t^{\beta}}\left(\frac{x^{\alpha}}{\alpha} \frac{\partial^{\alpha} v}{\partial x^{\alpha}}\right) \\
& +\zeta \frac{x^{\alpha}}{\alpha} u=\frac{x^{\alpha}}{\alpha} g(x, t) .
\end{aligned}
$$

(2) Applying (CDLT) on both sides of equation (51) and single conformable Laplace transform for equation (50), we get

$$
L_{x}^{\alpha} L_{t}^{\beta}\left[\frac{x^{\alpha}}{\alpha} \frac{\partial^{\beta} u}{\partial t^{\beta}}\right]=L_{x}^{\alpha} L_{t}^{\beta}\left[\frac{\partial^{\alpha}}{\partial x^{\alpha}}\left(\frac{x^{\alpha}}{\alpha} \frac{\partial^{\alpha} u}{\partial x^{\alpha}}\right)\right.
$$

$$
\begin{aligned}
& \left.+\frac{\partial^{\alpha+\beta}}{\partial x^{\alpha} \partial t \beta}\left(\frac{x^{\alpha}}{\alpha} \frac{\partial^{\alpha} u}{\partial x^{\alpha}}\right)-\zeta \frac{x^{\alpha}}{\alpha} v+\frac{x^{\alpha}}{\alpha} f(x, t)\right], \\
L_{x}^{\alpha} L_{t}^{\beta}\left[\frac{x^{\alpha}}{\alpha} \frac{\partial^{\beta} v}{\partial t^{\beta}}\right]= & L_{x}^{\alpha} L_{t}^{\beta}\left[\frac{\partial^{\alpha}}{\partial x^{\alpha}}\left(\frac{x^{\alpha}}{\alpha} \frac{\partial^{\alpha} v}{\partial x^{\alpha}}\right)+\frac{\partial^{\alpha+\beta}}{\partial x^{\alpha} \partial t^{\beta}}\left(\frac{x^{\alpha}}{\alpha} \frac{\partial^{\alpha} v}{\partial x^{\alpha}}\right)\right. \\
& \left.-\zeta \frac{x^{\alpha}}{\alpha} u+\frac{x^{\alpha}}{\alpha} g(x, t)\right] .
\end{aligned}
$$

On using Theorem 1 and Theorem 2, we obtain 


$$
\begin{aligned}
\frac{\mathrm{d}}{\mathrm{d} p} U(p, s)= & \frac{1}{s} \frac{\mathrm{d}}{\mathrm{d} p} L_{x}^{\alpha}\left[f_{1}(x)\right]-\frac{1}{s} L_{x}^{\alpha} L_{t}^{\beta}\left[\frac{\partial^{\alpha}}{\partial x^{\alpha}}\left(\frac{x^{\alpha}}{\alpha} \frac{\partial^{\alpha}}{\partial x^{\alpha}} u\right)\right. \\
& \left.+\frac{\partial^{\alpha+\beta}}{\partial x^{\alpha} \partial t^{\beta}}\left(\frac{x^{\alpha}}{\alpha} \frac{\partial^{\alpha}}{\partial x^{\alpha}} u\right)\right]-\zeta \frac{x^{\alpha}}{\alpha} v+\frac{1}{s} \frac{\mathrm{d}}{\mathrm{d} p}\left[L_{x}^{\alpha} L_{t}^{\beta}[f(x, t)]\right] \\
\frac{\mathrm{d}}{\mathrm{d} p} V(p, s)= & \frac{1}{s} \frac{\mathrm{d}}{\mathrm{d} p} L_{x}^{\alpha}\left[g_{1}(x)\right]-\frac{1}{s} L_{x}^{\alpha} L_{t}^{\beta}\left[\frac{\partial^{\alpha}}{\partial x^{\alpha}}\left(\frac{x^{\alpha}}{\alpha} \frac{\partial^{\alpha}}{\partial x^{\alpha}} v\right)\right. \\
& \left.+\frac{\partial^{\alpha+\beta}}{\partial x^{\alpha} \partial t^{\beta}}\left(\frac{x^{\alpha}}{\alpha} \frac{\partial^{\alpha}}{\partial x^{\alpha}} v\right)\right]-\zeta \frac{x^{\alpha}}{\alpha} u+\frac{1}{s} \frac{\mathrm{d}}{\mathrm{d} p}\left[L_{x}^{\alpha} L_{t}^{\beta}[g(x, t)]\right]
\end{aligned}
$$$$
\text { (3) By integrating both sides of equation (53) from } 0 \text { to } p
$$$$
\text { with respect to } p \text {, we have }
$$

$$
\begin{aligned}
& U(\mathrm{p}, \mathrm{s})=\frac{1}{s} \int_{0}^{p}\left(\frac{\mathrm{d}}{\mathrm{d} p} L_{x}^{\alpha}\left[f_{1}(x)\right]\right) \mathrm{d} p-\frac{1}{s} \int_{0}^{p} L_{x}^{\alpha} L_{t}^{\beta}\left[\frac{\partial^{\alpha}}{\partial x^{\alpha}}\left(\frac{x^{\alpha}}{\alpha} \frac{\partial^{\alpha}}{\partial x^{\alpha}} u\right)+\frac{\partial^{\alpha+\beta}}{\partial x^{\alpha} \partial t^{\beta}}\left(\frac{x^{\alpha}}{\alpha} \frac{\partial^{\alpha}}{\partial x^{\alpha}} u\right)-\zeta \frac{x^{\alpha}}{\alpha} v\right] \mathrm{d} p+\frac{1}{s} \int_{0}^{p}\left(\frac{\mathrm{d}}{\mathrm{d} p}\left[L_{x}^{\alpha} L_{t}^{\beta}[f(x, t)]\right]\right) \mathrm{d} p, \\
& V(\mathrm{p}, \mathrm{s})=\frac{1}{s} \int_{0}^{p}\left(\frac{\mathrm{d}}{\mathrm{d} p} L_{x}^{\alpha}\left[g_{1}(x)\right]\right) \mathrm{d} p-\frac{1}{s} \int_{0}^{p} L_{x}^{\alpha} L_{t}^{\beta}\left[\frac{\partial^{\alpha}}{\partial x^{\alpha}}\left(\frac{x^{\alpha}}{\alpha} \frac{\partial^{\alpha}}{\partial x^{\alpha}} v\right)+\frac{\partial^{\alpha+\beta}}{\partial x^{\alpha} \partial t^{\beta}}\left(\frac{x^{\alpha}}{\alpha} \frac{\partial^{\alpha}}{\partial x^{\alpha}} v\right)-\zeta \frac{x^{\alpha}}{\alpha} u\right] \mathrm{d} p+\frac{1}{s} \int_{0}^{p}\left(\frac{\mathrm{d}}{\mathrm{d} p}\left[L_{x}^{\alpha} L_{t}^{\beta}[g(x, t)]\right]\right) \mathrm{d} p .
\end{aligned}
$$

By applying double inverse Laplace transform for equation (54), we have

$$
\begin{aligned}
u(x, t)= & f_{1}(x)+L_{p}^{-1} L_{s}^{-1}\left[\frac{1}{s} \int_{0}^{p}\left(\frac{\mathrm{d}}{\mathrm{d} p}\left(L_{x}^{\alpha} L_{t}^{\beta}[f(x, t)]\right)\right) \mathrm{d} p\right]-L_{p}^{-1} L_{s}^{-1}\left[\frac{1}{s} \int_{0}^{p}\left(L_{x}^{\alpha} L_{t}^{\beta}\left[\frac{\partial^{\alpha}}{\partial x^{\alpha}}\left(\frac{x^{\alpha}}{\alpha} \frac{\partial^{\alpha} u}{\partial x^{\alpha}}\right)\right]\right) \mathrm{d} p\right] \\
& -L_{p}^{-1} L_{s}^{-1}\left[\frac{1}{s} \int_{0}^{p}\left(L_{x}^{\alpha} L_{t}^{\beta}\left[\frac{\partial^{\alpha+\beta}}{\partial x^{\alpha} \partial t^{\beta}}\left(\frac{x^{\alpha}}{\alpha} \frac{\partial^{\alpha} u}{\partial x^{\alpha}}\right)-\zeta \frac{x^{\alpha}}{\alpha} v\right]\right) \mathrm{d} p\right], \\
v(x, t)= & g_{1}(x)+L_{p}^{-1} L_{s}^{-1}\left[\frac{1}{s} \int_{0}^{p}\left(\frac{\mathrm{d}}{\mathrm{d} p}\left(L_{x}^{\alpha} L_{t}^{\beta}[g(x, t)]\right)\right) \mathrm{d} p\right]-L_{p}^{-1} L_{s}^{-1}\left[\frac{1}{s} \int_{0}^{p}\left(L_{x}^{\alpha} L_{t}^{\beta}\left[\frac{\partial^{\alpha}}{\partial x^{\alpha}}\left(\frac{x^{\alpha}}{\alpha} \frac{\partial^{\alpha} v}{\partial x^{\alpha}}\right)\right]\right) \mathrm{d} p\right] \\
& -L_{p}^{-1} L_{s}^{-1}\left[\frac{1}{s} \int_{0}^{p}\left(L_{x}^{\alpha} L_{t}^{\beta}\left[\frac{\partial^{\alpha+\beta}}{\partial x^{\alpha} \partial t^{\beta}}\left(\frac{x^{\alpha}}{\alpha} \frac{\partial^{\alpha} v}{\partial x^{\alpha}}\right)-\zeta \frac{x^{\alpha}}{\alpha} u\right]\right) \mathrm{d} p\right] .
\end{aligned}
$$

The (CDLTDM) defines the solutions of conformable coupled pseudoparabolic equations as $u(x, t)$ and $v(x, t)$ by the infinite series:

$$
\begin{aligned}
& u(x, t)=\sum_{n=0}^{\infty} u_{n}(x, t), \\
& v(x, t)=\sum_{n=0}^{\infty} v_{n}(x, t) .
\end{aligned}
$$

By substituting equation (57) into equations (55) and (56), we get

(4) Working with the double Laplace transform on both sides of equation (25) and using equation (26), we receive 


$$
\begin{aligned}
& \sum_{n=0}^{\infty} u_{n}(x, t)=f_{1}(x)+L_{p}^{-1} L_{s}^{-1}\left[\frac{1}{s} \int_{0}^{p}\left(\frac{\mathrm{d}}{\mathrm{d} p}\left(L_{x}^{\alpha} L_{t}^{\beta}[f(x, t)]\right)\right) \mathrm{d} p\right]-L_{p}^{-1} L_{s}^{-1}\left[\frac{1}{s} \int_{0}^{p}\left(L_{x}^{\alpha} L_{t}^{\beta}\left[\frac{\partial^{\alpha}}{\partial x^{\alpha}}\left(\frac{x^{\alpha}}{\alpha} \frac{\partial^{\alpha}}{\partial x^{\alpha}}\left(\sum_{n=0}^{\infty} u_{n}\right)\right)\right]\right) \mathrm{d} p\right] \\
& -L_{p}^{-1} L_{s}^{-1}\left[\frac{1}{s} \int_{0}^{p}\left(L_{x}^{\alpha} L_{t}^{\beta}\left[\frac{\partial^{\alpha+\beta}}{\partial x^{\alpha} \partial t^{\beta}}\left(\frac{x^{\alpha}}{\alpha} \frac{\partial^{\alpha}}{\partial x^{\alpha}}\left(\sum_{n=0}^{\infty} u_{n}\right)\right)\right]\right) \mathrm{d} p\right]+L_{p}^{-1} L_{s}^{-1}\left[\frac{1}{s} L_{x} L_{t}\left[\zeta \frac{x^{\alpha}}{\alpha} \sum_{n=0}^{\infty} v_{n}\right] \mathrm{d} p\right] \\
& \sum_{n=0}^{\infty} v_{n}(x, t)=g_{1}(x)+L_{p}^{-1} L_{s}^{-1}\left[\frac{1}{s} \int_{0}^{p}\left(\frac{\mathrm{d}}{\mathrm{d} p}\left(L_{x}^{\alpha} L_{t}^{\beta}[g(x, t)]\right)\right) \mathrm{d} p\right]-L_{p}^{-1} L_{s}^{-1}\left[\frac{1}{s} \int_{0}^{p}\left(L_{x}^{\alpha} L_{t}^{\beta}\left[\frac{\partial^{\alpha}}{\partial x^{\alpha}}\left(\frac{x^{\alpha}}{\alpha} \frac{\partial^{\alpha}}{\partial x^{\alpha}}\left(\sum_{n=0}^{\infty} v_{n}\right)\right)\right]\right) \mathrm{d} p\right] \\
& -L_{p}^{-1} L_{s}^{-1}\left[\frac{1}{s} \int_{0}^{p}\left(L_{x}^{\alpha} L_{t}^{\beta}\left[\frac{\partial^{\alpha+\beta}}{\partial x^{\alpha} \partial t^{\beta}}\left(\frac{x^{\alpha}}{\alpha} \frac{\partial^{\alpha}}{\partial x^{\alpha}}\left(\sum_{n=0}^{\infty} v_{n}\right)\right)\right]\right) \mathrm{d} p\right]+L_{p}^{-1} L_{s}^{-1}\left[\frac{1}{s} L_{x} L_{t}\left[\zeta \frac{x^{\alpha}}{\alpha} \sum_{n=0}^{\infty} u_{n}\right] \mathrm{d} p\right] \text {. }
\end{aligned}
$$

This technique suggests that the zeroth components $u_{0}$ and $v_{0}$ are identified by the initial conditions and from source terms as follows:

$$
\begin{aligned}
& u_{0}=f_{1}(x)+L_{p}^{-1} L_{s}^{-1}\left[\frac{1}{s} \int_{0}^{p}\left(\frac{\mathrm{d}}{\mathrm{d} p}\left(L_{x}^{\alpha} L_{t}^{\beta}[f(x, t)]\right)\right) \mathrm{d} p\right], \\
& v_{0}=g_{1}(x)+L_{p}^{-1} L_{s}^{-1}\left[\frac{1}{s} \int_{0}^{p}\left(\frac{\mathrm{d}}{\mathrm{d} p}\left(L_{x}^{\alpha} L_{t}^{\beta}[g(x, t)]\right)\right) \mathrm{d} p\right] .
\end{aligned}
$$

$$
\begin{aligned}
u_{n+1}= & -L_{p}^{-1} L_{s}^{-1}\left[\frac{1}{s} \int_{0}^{p}\left(L_{x}^{\alpha} L_{t}^{\beta}\left[\frac{\partial^{\alpha}}{\partial x^{\alpha}}\left(\frac{x^{\alpha}}{\alpha} \frac{\partial^{\alpha} u_{n}}{\partial x^{\alpha}}\right)\right]\right) \mathrm{d} p\right]-L_{p}^{-1} L_{s}^{-1}\left[\frac{1}{s} \int_{0}^{p}\left(L_{x}^{\alpha} L_{t}^{\beta}\left[\frac{\partial^{\alpha+\beta}}{\partial x^{\alpha} \partial t^{\beta}}\left(\frac{x^{\alpha}}{\alpha} \frac{\partial^{\alpha} u_{n}}{\partial x^{\alpha}}\right)\right]\right) \mathrm{d} p\right]+L_{p}^{-1} L_{s}^{-1}\left[\frac{1}{s} L_{x} L_{t}\left[\zeta \frac{x^{\alpha}}{\alpha} v_{n}\right] \mathrm{d} p\right], \\
v_{n+1}= & -L_{p}^{-1} L_{s}^{-1}\left[\frac{1}{s} \int_{0}^{p}\left(L_{x}^{\alpha} L_{t}^{\beta}\left[\frac{\partial^{\alpha}}{\partial x^{\alpha}}\left(\frac{x^{\alpha}}{\alpha} \frac{\partial^{\alpha} v_{n}}{\partial x^{\alpha}}\right)\right]\right) \mathrm{d} p\right] \\
& -L_{p}^{-1} L_{s}^{-1}\left[\frac{1}{s} \int_{0}^{p}\left(L_{x}^{\alpha} L_{t}^{\beta}\left[\frac{\partial^{\alpha+\beta}}{\partial x^{\alpha} \partial t^{\beta}}\left(\frac{x^{\alpha}}{\alpha} \frac{\partial^{\alpha} v_{n}}{\partial x^{\alpha}}\right)\right]\right) \mathrm{d} p\right]+L_{p}^{-1} L_{s}^{-1}\left[\frac{1}{s} L_{x} L_{t}\left[\zeta \frac{x^{\alpha}}{\alpha} u_{n}\right] \mathrm{d} p\right] .
\end{aligned}
$$

In order to ensure the four techniques for solving the conformable fractional coupled pseudoparabolic equation, we will consider the following example.

Example 5. Consider the following homogeneous form of conformable coupled pseudoparabolic equations:

$$
\begin{aligned}
& \frac{\partial^{\beta} u}{\partial t^{\beta}}-2 \frac{\alpha}{x^{\alpha}} \frac{\partial^{\alpha}}{\partial x^{\alpha}}\left(\frac{x^{\alpha}}{\alpha} \frac{\partial^{\alpha} u}{\partial x^{\alpha}}\right)-\frac{\alpha}{x^{\alpha}} \frac{\partial^{\alpha+\beta}}{\partial x^{\alpha} \partial t^{\beta}}\left(\frac{x^{\alpha}}{\alpha} \frac{\partial^{\alpha} u}{\partial x^{\alpha}}\right)+2 v=0, \\
& \frac{\partial^{\beta} v}{\partial t^{\beta}}-2 \frac{\alpha}{x^{\alpha}} \frac{\partial^{\alpha}}{\partial x^{\alpha}}\left(\frac{x^{\alpha}}{\alpha} \frac{\partial^{\alpha} v}{\partial x^{\alpha}}\right)-\frac{\alpha}{x^{\alpha}} \frac{\partial^{\alpha+\beta}}{\partial x^{\alpha} \partial t^{\beta}}\left(\frac{x^{\alpha}}{\alpha} \frac{\partial^{\alpha} v}{\partial x^{\alpha}}\right)+2 u=0,
\end{aligned}
$$

where

$$
\begin{gathered}
0 \leq x<\infty, \\
0 \leq t<\infty, \\
0<\alpha \leq 1, \\
0<\beta \leq 1, \\
u(x, 0)=\left(\frac{x^{\alpha}}{\alpha}\right)^{2}, \\
v(x, 0)=\left(\frac{x^{\alpha}}{\alpha}\right)^{2} .
\end{gathered}
$$

with conditions

By applying the above method and Theorem 1 for equation (62) and (64) and using equations (59), (60), and (61), we obtain 


$$
\begin{aligned}
& u_{0}=\left(\frac{x^{\alpha}}{\alpha}\right)^{2} \\
& v_{0}=\left(\frac{x^{\alpha}}{\alpha}\right)^{2} \\
& u_{1}=-L_{p}^{-1} L_{s}^{-1}\left[\frac { 1 } { s } L _ { x } L _ { t } \left[2 \frac{\partial^{\alpha}}{\partial x^{\alpha}}\left(\frac{x^{\alpha}}{\alpha} \frac{\partial^{\alpha}}{\partial x^{\alpha}} u_{0}\right)\right.\right. \\
& \left.\left.+\frac{\partial^{\alpha+\beta}}{\partial x^{\alpha} \partial t^{\beta}}\left(\frac{x^{\alpha}}{\alpha} \frac{\partial^{\alpha}}{\partial x^{\alpha}} u_{0}\right)-2 \frac{x^{\alpha}}{\alpha} v_{0}\right]\right] \\
& =8 \frac{t^{\beta}}{\beta}-2\left(\frac{x^{\alpha}}{\alpha}\right)^{2} \frac{t^{\beta}}{\beta}, \\
& v_{1}=-L_{p}^{-1} L_{s}^{-1}\left[\frac { 1 } { s } L _ { x } L _ { t } \left[2 \frac{\partial^{\alpha}}{\partial x^{\alpha}}\left(\frac{x^{\alpha}}{\alpha} \frac{\partial^{\alpha}}{\partial x^{\alpha}} v_{0}\right)\right.\right. \\
& \left.\left.+\frac{\partial^{\alpha+\beta}}{\partial x^{\alpha} \partial t^{\beta}}\left(\frac{x^{\alpha}}{\alpha} \frac{\partial^{\alpha}}{\partial x^{\alpha}} v_{0}\right)-2 \frac{x^{\alpha}}{\alpha} u_{0}\right]\right] \\
& =8 \frac{t^{\beta}}{\beta}-2\left(\frac{x^{\alpha}}{\alpha}\right)^{2} \frac{t^{\beta}}{\beta} \\
& u_{2}=-L_{p}^{-1} L_{s}^{-1}\left[\frac { 1 } { s } L _ { x } L _ { t } \left[2 \frac{\partial^{\alpha}}{\partial x^{\alpha}}\left(\frac{x^{\alpha}}{\alpha} \frac{\partial^{\alpha}}{\partial x^{\alpha}} u_{1}\right)\right.\right. \\
& \left.\left.+\frac{\partial^{\alpha+\beta}}{\partial x^{\alpha} \partial t^{\beta}}\left(\frac{x^{\alpha}}{\alpha} \frac{\partial^{\alpha}}{\partial x^{\alpha}} u_{1}\right)-2 \frac{x^{\alpha}}{\alpha} v_{1}\right]\right] \\
& =-16\left(\frac{t^{\beta}}{\beta}\right)^{2}-8 \frac{t^{\beta}}{\beta}+2\left(\frac{x^{\alpha}}{\alpha}\right)^{2}\left(\frac{t^{\beta}}{\beta}\right)^{2} \\
& v_{2}=-L_{p}^{-1} L_{s}^{-1}\left[\frac { 1 } { s } L _ { x } L _ { t } \left[2 \frac{\partial^{\alpha}}{\partial x^{\alpha}}\left(\frac{x^{\alpha}}{\alpha} \frac{\partial^{\alpha}}{\partial x^{\alpha}} v_{1}\right)\right.\right. \\
& \left.\left.+\frac{\partial^{\alpha+\beta}}{\partial x^{\alpha} \partial t^{\beta}}\left(\frac{x^{\alpha}}{\alpha} \frac{\partial^{\alpha}}{\partial x^{\alpha}} v_{1}\right)-2 \frac{x^{\alpha}}{\alpha} u_{1}\right]\right] \\
& =-16\left(\frac{t^{\beta}}{\beta}\right)^{2}-8 \frac{t^{\beta}}{\beta}+2\left(\frac{x^{\alpha}}{\alpha}\right)^{2}\left(\frac{t^{\beta}}{\beta}\right)^{2}, \\
& u_{3}=16\left(\frac{t^{\beta}}{\beta}\right)^{3}+16\left(\frac{t^{\beta}}{\beta}\right)^{2}-\frac{4}{3}\left(\frac{x^{\alpha}}{\alpha}\right)^{2}\left(\frac{t^{\beta}}{\beta}\right)^{3} \text {, } \\
& v_{3}=16\left(\frac{t^{\beta}}{\beta}\right)^{3}+16\left(\frac{t^{\beta}}{\beta}\right)^{2}-\frac{4}{3}\left(\frac{x^{\alpha}}{\alpha}\right)^{2}\left(\frac{t^{\beta}}{\beta}\right)^{3} \text {, } \\
& u_{4}=-\frac{32}{3}\left(\frac{t^{\beta}}{\beta}\right)^{4}-16\left(\frac{t^{\beta}}{\beta}\right)^{3}+\frac{2}{3}\left(\frac{x^{\alpha}}{\alpha}\right)^{2}\left(\frac{t^{\beta}}{\beta}\right)^{4} \text {, } \\
& v_{4}=-\frac{32}{3}\left(\frac{t^{\beta}}{\beta}\right)^{4}-16\left(\frac{t^{\beta}}{\beta}\right)^{3}+\frac{2}{3}\left(\frac{x^{\alpha}}{\alpha}\right)^{2}\left(\frac{t^{\beta}}{\beta}\right)^{4} \text {, }
\end{aligned}
$$

and similarly for the rest components. Using equation (57), the series solutions are therefore given by

$$
\begin{aligned}
& u=u_{0}+u_{1}+u_{2}+\cdots=\left(1-\left(2 \frac{t^{\beta}}{\beta}\right)+\frac{\left(2\left(t^{\beta} / \beta\right)\right)^{2}}{2 !}\right. \\
& \left.-\frac{\left(2\left(t^{\beta} / \beta\right)\right)^{3}}{3 !}+\frac{\left(2\left(t^{\beta} / \beta\right)\right)^{4}}{4 !}-\cdots\right)\left(\frac{x^{\alpha}}{\alpha}\right)^{2}, \\
& v=v_{0}+v_{1}+v_{2}+\cdots=\left(1-\left(2 \frac{t^{\beta}}{\beta}\right)+\frac{\left(2\left(t^{\beta} / \beta\right)\right)^{2}}{2 !}\right. \\
& \left.-\frac{\left(2\left(t^{\beta} / \beta\right)\right)^{3}}{3 !}+\frac{\left(2\left(t^{\beta} / \beta\right)\right)^{4}}{4 !}-\cdots\right)\left(\frac{x^{\alpha}}{\alpha}\right)^{2} .
\end{aligned}
$$

Hence,

$$
\begin{aligned}
& u(x, t)=\left(\frac{x^{\alpha}}{\alpha}\right)^{2} e^{-2\left(t^{\beta} / \beta\right),} \\
& v(x, t)=\left(\frac{x^{\alpha}}{\alpha}\right)^{2} e^{-2\left(t^{\beta} / \beta\right)}
\end{aligned}
$$

The exact solution is obtained by taking $\alpha=1$ and $\beta=1$, as follows:

$$
\begin{aligned}
& u(x, t)=x^{2} e^{-2 t}, \\
& v(x, t)=x^{2} e^{-2 t} .
\end{aligned}
$$

\section{Numerical Result}

In this section, we discuss the precision and efficiency of the (CDLTDM) by numerical results of $u(x, t)$ for the exact solution when $(\alpha=\beta=1)$ and approximate solutions at $\alpha$ and $\beta$ taking different fractional values for conformable pseudoparabolic equation and conformable coupled pseudoparabolic equation. The solutions of equation (30) are depicted in Figures 1 and 2, respectively.

In Figure 1, the approximate solutions of equation (30) at $(t=1)$ and $(\alpha=\beta)$, taking different fractional values, are compared and we found that the numerical solution becomes close to the exact solution when the fractional value increases:

$$
(\alpha=\beta=1)
$$

Figure 2 indicates that the exact solution at $(\alpha \beta=1)$ of equation (30) and the approximate solution of equation (30) decrease at the fractional derivative values $(\alpha 0.99)$ and $(\beta 0.98,0.96,0.94)$. Similarly, the exact solution and approximate solution of equation (44) are demonstrated in 


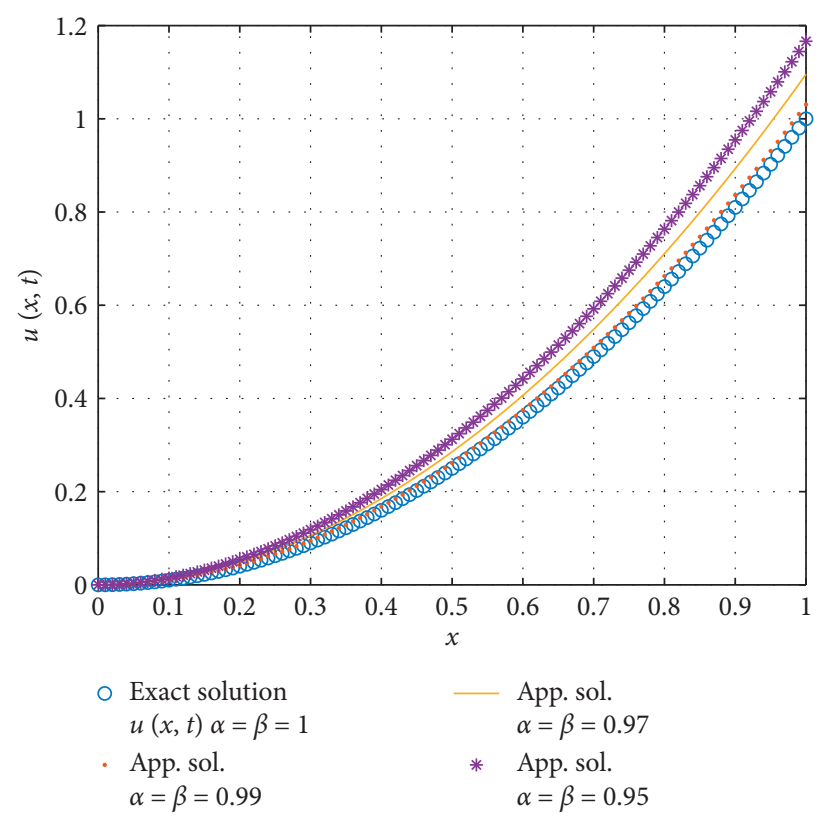

FIgURE 1: The exact and approximate solutions of $u(x, t)$ for Example 3, when $\alpha=\beta$.

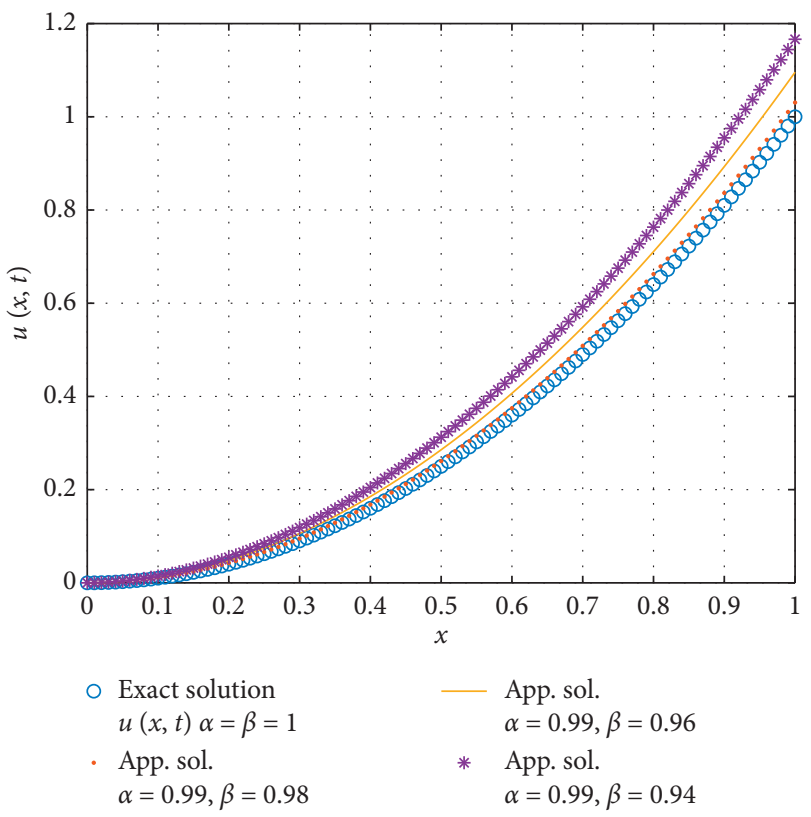

FIGURE 2: The exact and approximate solutions of $u(x, t)$, for Example 3, when we take different values of fractional order $\beta$ and $\alpha=0.99$.

Figures 3 and 4 . Figure 3 give the plots of the behaviour of equation (44) when $(t=1)$ and $(\alpha=\beta)$ with different fractional values taken in this case; the solution $u(x, t)$ becomes close to the exact solution at $(\alpha=\beta)$ close to one.

Figure 4 shows the approximate solution of equation (44) with $(0<\alpha \leq 1),(\beta=0.99)$, and $(t=1)$; in such a case,

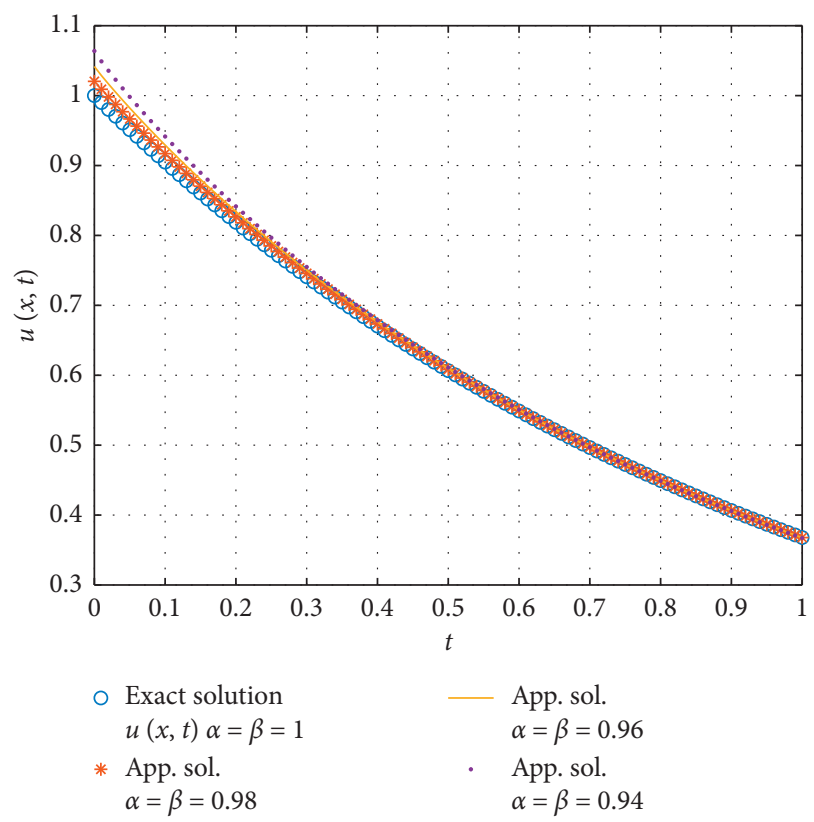

FIgURE 3: The exact and approximate solutions of $u(x, t)$ for Example 4, when $\alpha=\beta$.

the function $u(x, t)$ gradually decreases. Finally, Figure 5 suggests that in the solutions of equation (62) at $(t=1)$ and $(0<\alpha=\beta \leq 1)$, we find that the numerical solution becomes close to the exact solution when the fractional value increases.

Figure 6 demonstrates that the exact solution at $(\alpha=$ $\beta=1$ ) of equation (62) and the approximate solution of 


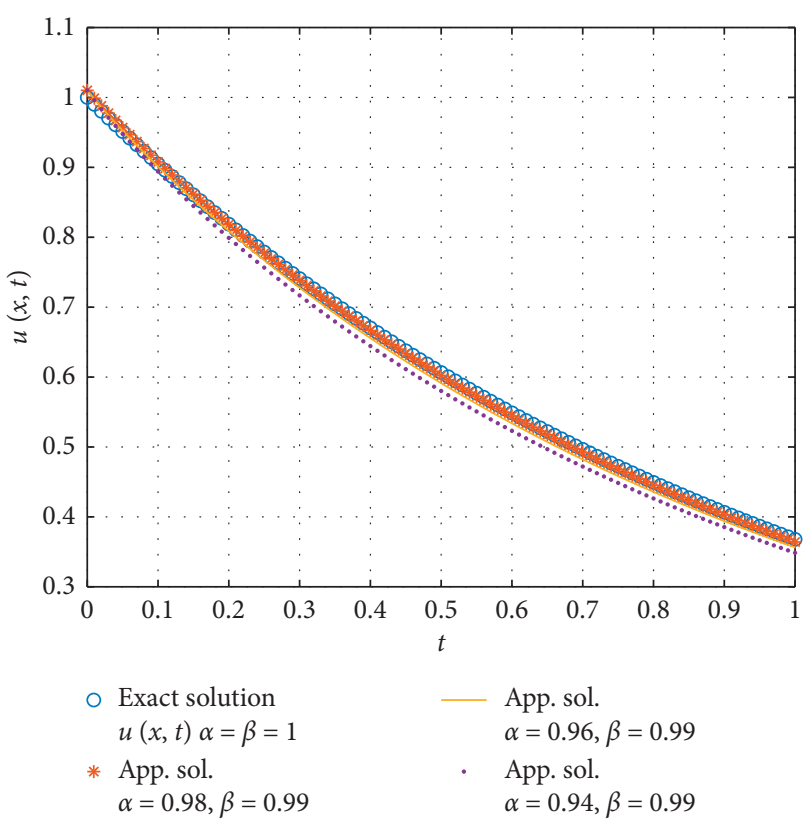

Figure 4: The exact and approximate solutions of $u(x, t)$, for Example 4, when we take different values of fractional order $\alpha$ and $\beta=0.99$.

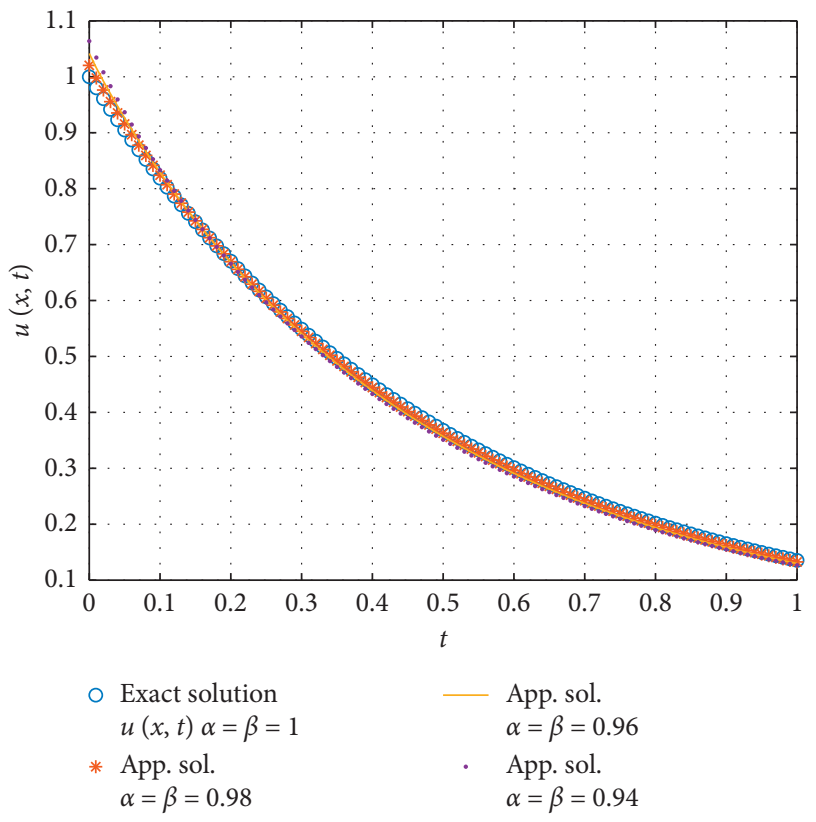

FIgURE 5: The exact and approximate solutions of $u(x, t)$ for Example 5, when $\alpha=\beta$.

equation (62) are concave upward at the fractional derivative increasing when $(0<\alpha \leq 1)$ and $(\beta)$ fixed.

\section{Conclusion}

In this work, singular one-dimensional conformable pseudoparabolic equation and conformable coupled pseudoparabolic equation have been considered. Then, new

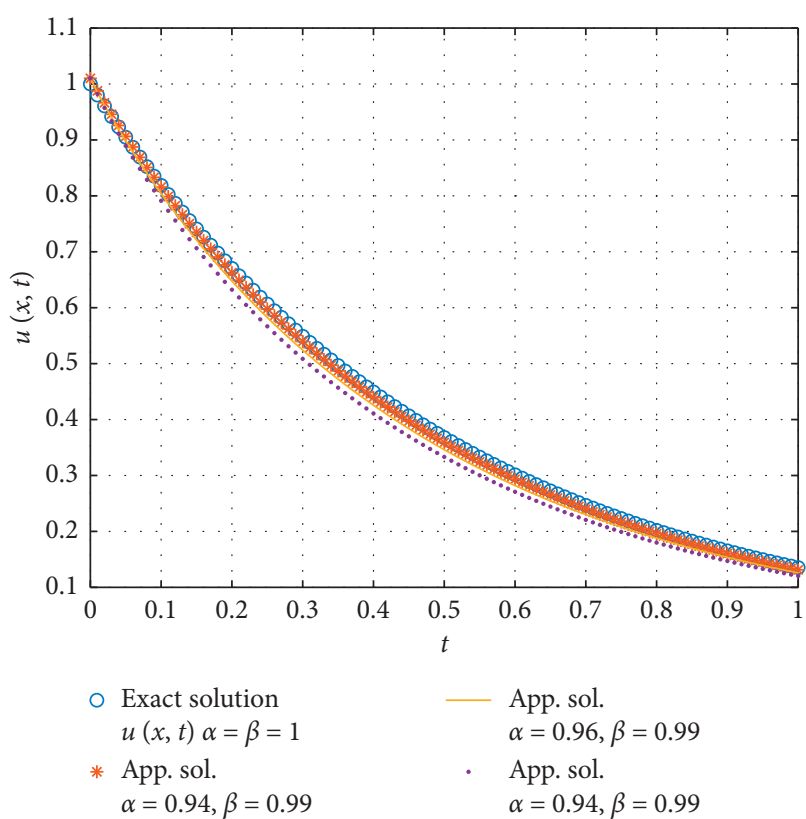

FIgURE 6: The exact and approximate solutions of $u(x, t)$, for Example 5, when we take different values of fractional order $\alpha$ and $\beta=0.99$.

conformable double Laplace transform decomposition methods have been applied to the problems. Finally, we gave three differential examples to show that this method is applicable and valid. The suggested method can also be applied for systems with more than two linear and nonlinear partial differential equations. In addition, if we let $\alpha=1$ and $\beta=1$ in Examples 3 and 4, we get the solution which is considered in [22]. All figure results are obtained by using Matlab.

\section{Data Availability}

No data were used to support this study.

\section{Conflicts of Interest}

The authors declare that they have no conflicts of interest.

\section{Acknowledgments}

The authors would like to extend their sincere appreciation to the Deanship of Scientific Research at King Saud University for funding this research group (RG-1440-030).

\section{References}

[1] S. Gala and M. A. Ragusa, "A regularity criterion for 3D micropolar fluid flows in terms of one partial derivative of the velocity," Annales Polonici Mathematici, vol. 116, no. 3, pp. 217-228, 2016.

[2] Q. Qi, Y. Chen, and Q. Wang, "Blow-up phenomena for a pseudo-parabolic system with variable exponents," Electronic Journal of Qualitative Theory of Differential Equations, vol. 36, no. 36, pp. 1-9, 2017. 
[3] M. A. Ragusa, "Commutators of fractional integral operators in Vanishing-Morrey Spaces," Journal of Global Optimization, vol. 40, no. 1-3, pp. 361-368, 2008.

[4] Y. Zheng and Z. B. Fang, "Qualitative properties for a pseudoparabolic equation with nonlocal reaction term," Boundary Value Problems, vol. 2019, no. 1, p. 134, 2019.

[5] F. Wu and X.-J. Yang, "Approximate solution of the nonlinear diffusion equation of multiple orders," Thermal Science, vol. 20 , no. 3, pp. 683-687, 2016.

[6] S.-P. Yan, W.-P. Zhong, and X.-J. Yang, "A novel series method for fractional diffusion equation within Caputo fractional derivative," Thermal Science, vol. 20, no. 3, pp. 695-699, 2016.

[7] T. Abdeljawad, "On conformable fractional calculus," Journal of Computational and Applied Mathematics, vol. 279, pp. 57-66, 2015.

[8] R. Khalil, M. Al Horani, A. Yousef, and M. Sababheh, "A new definition of fractional derivative," Journal of Computational and Applied Mathematics, vol. 264, pp. 65-70, 2014.

[9] U. N. Katugampola, "A new fractional derivative with classical properties,” 2014, https://arxiv.org/abs/1410.6535.

[10] U. N. Katugampola, "Mellin transforms of generalized fractional integrals and derivatives," Applied Mathematics and Computation, vol. 257, pp. 566-580, 2015.

[11] A. Gökdogan, E. Ünal, and E. Celik, "Existence and uniqueness theorems for sequential linear conformable fractional differential equations," Miskolc Mathematical Notes, vol. 17, no. 1, pp. 267-279, 2016.

[12] M. D. Ortigueira and J. A. Tenreiro Machado, "What is a fractional derivative?" Journal of Computational Physics, vol. 293, pp. 4-13, 2015.

[13] Z. Rahimi, G. Rezazadeh, W. Sumelka, and X.-J. Yang, "A study of critical point instability of micro and nano beams under a distributed variable-pressure force in the framework of the inhomogeneous non-linear nonlocal theory," Archives of Mechanics, vol. 69, no. 6, pp. 413-433, 2017.

[14] Z. Rahimi, W. Sumelka, and X.-J. Yang, "A new fractional nonlocal model and its application in free vibration of Timoshenko and Euler-Bernoulli beams," The European Physical Journal Plus, vol. 132, no. 11, p. 479, 2017.

[15] F. Jarada and T. Abdeljawadb, "A modified Laplace transform for certain generalized fractional operators," Results in Nonlinear Analysis, vol. 1, no. 2, pp. 88-98, 2018.

[16] O. Özkan and K. Ali, "The analytical solutions for conformable integral equations and integro-differential equations by conformable Laplace transform," Optical and Quantum Electronics, vol. 50, no. 2, p. 81, 2018.

[17] A. Korkmaz and K. Hosseini, "Exact solutions of a nonlinear conformable time-fractional parabolic equation with exponential nonlinearity using reliable methods," Optical and Quantum Electronics, vol. 49, no. 8, p. 278, 2017.

[18] H. Thabet and S. Kendre, "Analytical solutions for conformable space-time fractional partial differential equations via fractional differential transform," Chaos, Solitons \& Fractals, vol. 109, pp. 238-245, 2018.

[19] M. S. Hashemi, "Invariant subspaces admitted by fractional differential equations with conformable derivatives," Chaos, Solitons \& Fractals, vol. 107, pp. 161-169, 2018.

[20] B. Mohamed, H. Khalid, and M. Said, "Sequential evolution conformable differential equations of second order with nonlocal condition," Advances in Difference Equations, vol. 2019, no. 1, p. 21, 2019.
[21] O. Özkan and K. Ali, "On conformable double Laplace transform," Optical and Quantum Electronics, vol. 50, no. 2, p. 103, 2018.

[22] H. E. Gadain, "Solving coupled pseudo-parabolic equation using a modified double Laplace decomposition method," Acta Mathematica Scientia, vol. 38, no. 1, pp. 333-346, 2018. 